Acute effects of a guided relaxation routine (body scan) on tobacco withdrawal symptoms and cravings in abstinent smokers

Pages: 15

Word count: 2771

Mark Cropley $^{1,}$ Michael Ussher $^{2}$, Elli Charitou ${ }^{1}$

Department of Psychology, University of Surrey, Guildford, Surrey, GU2 7XH, UK ${ }^{1}$

Division of Community Health Sciences, St.George's, University of London, London, SW17 0RE, UK ${ }^{2}$

Correspondence to:

Dr Mark Cropley

Department of Psychology

University of Surrey

Guildford

Surrey

GU2 7XH, UK

Tel: +44 (0) 1483686928

Fax: + +44 1483689553

E-mail: mark.cropley@surrey.ac.uk 


\title{
Acute effects of a guided relaxation routine (body scan) on tobacco withdrawal symptoms and cravings in abstinent smokers
}

\author{
Aims To examine the acute effects of a guided relaxation routine (body scan) on \\ desire to smoke and tobacco withdrawal symptoms in overnight abstinent smokers. \\ Design Experimental.
}

Participants Thirty individuals reporting to smoke 10 or more cigarettes daily for at least three years.

Intervention Participants were randomly assigned to complete a 10 minute body scan (experimental group $n=15$ ) or listen to a natural history passage for 10 minutes (control group $n=15$ ).

Measurement Ratings of strength of desire to smoke and smoking withdrawal symptoms were assessed at baseline, immediately after the interventions, and 5, 10 \& 15 minutes post-intervention.

Findings There was a significant group by time interaction for strength of desire to smoke. The mean desire to smoke rating was significantly lower in the body scan group relative to the control group immediately after the intervention, and 5 minutes post intervention. The body scan group also reported lower ratings of irritability, tension, and restlessness, relative to the controls.

Conclusion A brief body scan intervention reduces strength of desire to smoke and some tobacco withdrawal symptoms in temporarily abstaining smokers. The body scan may be beneficial as a technique for managing cigarette cravings and withdrawal.

Keywords smoking cessation, body scan, mindfulness, withdrawal 


\section{INTRODUCTION}

Severe cravings for cigarettes are a reliable predictor of smoking relapse (1). Nicotine replacement therapy (NRT) and bupropion have been shown to be two of the most effective aids to smoking cessation $(2 ; 3)$ and these therapies are thought to work largely through moderating cigarette cravings and withdrawal symptoms. Laboratory studies have shown that non-pharmaceutical interventions; for example, cardiovascular exercise (4) and glucose consumption (5), can also be effective for reducing cigarette cravings and withdrawal symptoms. However, exercise is impractical in many situations and glucose consumption may not be appropriate for some individuals (e.g., diabetics). Further behavioural and cognitive strategies, that are appealing to the majority of smokers, need to be developed for managing cigarette cravings.

Stress is likely to exacerbate the desire to smoke, to motivate smoking, and stress has been associated with smoking relapse (6-9). Stress reduction techniques may therefore be effective for managing cravings. One stress management technique that has been attracting research attention is mindfulness (10). Mindfulness emphasises the focussing of attention on the present, without questioning or being judgemental of the information that comes to mind. Mindfulness techniques have been incorporated in many clinical programmes and practising mindfulness has been shown to improve the quality of life in patients within a variety of clinical and non-clinical issues (11).

One aspect of mindfulness, which is the focus of the present study, is the 'body scan'. Body scan is a cognitive relaxation technique that requires individuals to move their focus of attention around different parts of their body. The individual first concentrates on observing their breathing, before moving their awareness to other body regions. Body scan has been found to be an effective stress management 
technique (10), and is therefore likely to have beneficial effects for reducing the urge to smoke. Body scan is relatively easy to learn, it can be used in a wide variety of situations and it may be particularly attractive among those smokers who cannot, or who choose not to, use NRT products, e.g. pregnant women, or those adverse to exercise or glucose.

A previous study observed no effect of a body scan on desire to smoke and withdrawal symptoms, compared with a passive control condition (12). However, in this study the participants were not guided through the body scan, as is generally advised. Therefore, the present study assessed the effects of a guided body scan, versus a neutral audio recording, on ratings of desire to smoke and cigarette withdrawal symptoms among smokers who abstained from smoking overnight. It was hypothesised that those undergoing the body scan would report a significant reduction in desire to smoke and cigarette withdrawal symptoms, relative to those in the control group.

\section{METHOD}

\section{Participants}

The sample included 18 men and 12 women aged $21-31$ years $($ Mean $(S D)=25.5$ (2.9) years), smoking at least 10 cigarettes per day for at least three consecutive years. Participants were recruited via posters, within the campus of the University of Surrey. 


\section{Materials}

\section{Body scan}

Typically, a body scan takes 30-45 minutes; however, for the purpose of the study a 10-minute version was developed, as this would be more appealing and practical for the majority of smokers. Once comfortable, individuals were asked to follow an audio recording of a guided body scan. First, they were instructed to focus on their breathing by concentrating attention on their abdominal area. Focus of awareness was then gradually moved to other areas of the body.

\section{Control}

The control condition involved participants listening to a 10-minute audio recording of a natural history text (13). Individuals who listened to a pilot audio reported this to be a neutral, but relaxing passage. Pauses within both recordings were made in approximately the same place. For both conditions, audio instructions were presented via headphones. A copy of the two recordings is available from the corresponding author.

\section{Measures}

Background measures covered smoking characteristics, including the Fagerström Test for Nicotine Dependence (FTND) (14) and demographic details. The FTND was used to assess self-reported dependency levels between the body scan and control participants. This is a widely used and valid measure of nicotine dependence (15). Seven cigarette withdrawal symptoms were assessed using the Mood and Physical Symptoms Scale (MPSS) (16). These were strength of desire to smoke, irritability, tension, depression, restlessness, difficulty concentrating, and stress. Each was rated on a 7 - point scale, for example, 'How strong is your desire to smoke right now?' or 'How tense to you feel right now?' $(1=$ not at all, $4=$ somewhat, $7=$ extremely $)$. The 
MPSS has been widely used to access transient shifts in cigarette withdrawal symptoms and has good psychometric properties $(17 ; 18)$.

\section{Procedure}

Volunteers were initially screened for carbon monoxide (CO) levels to confirm smoking status (all individuals reported $\mathrm{CO}$ concentrations $>15 \mathrm{ppm}$ ) and completed background measures. Following overnight abstinence, each individual attended a laboratory testing session between 12 noon and $2 \mathrm{pm}$. On entering the laboratory individuals were seated and their baseline $\mathrm{CO}$ concentrations were assessed to confirm smoking abstinence $(<10 \mathrm{ppm})$. Next, they gave baseline ratings for withdrawal symptoms and desire to smoke using the MPSS. Participants were then randomly assigned to either the body scan or control condition. Further ratings of the MPSS were taken immediately after the interventions, and at five, 10 and 15 minutes post-intervention.

\section{RESULTS}

There was no significant difference between the two groups for any of the demographic or smoking characteristics (Table 1) or any of the baseline MPSS items. For each MPSS item, baseline ratings were subtracted from ratings taken immediately after the intervention and at, five, 10 and 15 minutes post-intervention. Depression, irritability and stress, had very low baseline ratings and were therefore excluded from further analysis. Means and SD for all the MPSS items are available from either author. The remaining data was analysed using a series of group (body scan/control) by time $(0,5,10,15$ minutes post-intervention) repeated measures Analysis of Variance (ANOVA). Where the overall group by time interaction was significant, planned comparisons were calculated for baseline versus each further time point. For 
ease of understanding, means and the results of the ANOVA, plus effect size (partial $\mathrm{eta}^{2)}$ for all smoking withdrawal items are presented in Figure 1.

There were significant main group effects for irritability, tension, and restlessness. In all cases the body scan group gave a lower rating relative to the controls: Irritability (Mean -1.63 vs. -0.15 ); tension (Mean -2.01 vs. - 0.58), and restlessness (Mean -1.28 vs. -0.51). There was as expected a significant effect of time for strength of desire to smoke, irritability, tension, and restlessness. There was a significant group by time interaction for strength of desire to smoke. The mean strength of desire to smoke rating was 1 point lower (on a 7 point scale) in the body scan group relative to the control group immediately after the intervention, and 0.93 points lower at 5 minutes $(\mathrm{P}<0.05)$. There was no significant group by time interaction for irritability, restlessness or tension.

\section{DISCUSSION}

This study examined the effects of a brief body scan following overnight abstinence from smoking. Relative to baseline, strength of desire to smoke was reduced up to five minutes following the 10-minute body scan, compared with the control condition. This effect is modest, and requires replication but indicates that a brief body scan has the potential to provide relief for smokers experiencing severe cigarette cravings during smoking abstinence. The laboratory is an artificial setting for smokers and the effects of the body scan on smoking cravings may not be as powerful in this environment as it is in the real world. In the laboratory participants are required to sit quietly and do nothing between the rating periods whereas in daily life smokers would be able to distract themselves from their cravings by doing other things e.g., working. 
The body scan also showed benefits for moderating feelings of irritability, tension and restlessness that are common symptoms during smoking withdrawal. Therefore, the body scan may have broader benefits beyond the management of cigarette cravings. Overall these findings are comparable to other studies that have used similar methodology (19;20). For example, Daniel et al. (19) reported a reduction from baseline of 1.5 in ratings of 'strength of desire to smoke' following 10 minutes of moderate intensity exercise, and a reduction of approximately 1.5 for irritability, tension and restlessness, in the laboratory for smokers who had abstained from smoking between 11-15 hours.

The body scan could be readily incorporated into behaviorally-based stop smoking programmes or could be utilized by individuals who choose not to use professional smoking cessation services. The body scan does not need to be performed in a seated position and with practice the technique can be used without an audio aid. Therefore the body scan is potentially beneficial across the large majority of smokers; including those who are contraindicated for pharmaceutical aids or who prefer not to use such products (e.g. pregnant smokers).

It was beyond the scope of this study to investigate the mechanisms involved; however, it is possible that the effect of body scan was partly the result of distraction and could also be due to stress reduction effects. As reported above, stress has been shown to affect cigarette withdrawal symptoms and we expected participants in the body scan condition to report lower ratings of stress following the intervention. Unfortunately, due to low baseline ratings from both groups it was not possible to assess whether the intervention did indeed affect stress. We did nevertheless; find a greater reduction in tension, restlessness and irritability, which may be considered manifestations of stress, for the body scan group relative to the controls. Further 
studies are needed to explore mechanisms; for example, through using psychophysiological measures such as EEG and cortisol. In addition, research is needed to assess the benefits of the body scan when high levels of stress are reported. Studies are also needed in order to assess how body scan interacts with the effects of NRT; for example, does body scan have an additive or synergistic effect when combined with NRT? In addition, work is needed to compare the body scan with other stress management techniques; for example, with different deep breathing exercises. Finally, this study was limited to temporarily abstinent smokers in a laboratory setting and research is required to explore whether the technique is similarly effective outside the laboratory, or during an actual quit attempt and with repeated usage.

Notwithstanding, the present results suggest that training individuals to use the body scan relaxation technique might be beneficial for some people as a strategy for managing the urge to smoke. 


\section{References}

(1) West RJ, Hajek P, Belcher M. Severity of withdrawal symptoms as a predictor of outcome of an attempt to quit smoking. Psychol Med 1989;19:981-5.

(2) Silagy C, Lancaster T, Stead L, Mant D, Fowler G. Nicotine replacement therapy for smoking cessation. Cochrane Database Syst Rev 2004; (3):CD000146.

(3) Hughes J, Stead L, Lancaster T. Antidepressants for smoking cessation. Cochrane Database Syst Rev 2007; 1:CD000031.

(4) Ussher M, Nunziata P, Cropley M, West R. Effect of a short bout of exercise on tobacco withdrawal symptoms and desire to smoke. Psychopharmacology (Berl) 2001; 158:66-72.

(5) West R, Courts S, Beharry S, May S, Hajek P. Acute effect of glucose tablets on desire to smoke. Psychopharmacology (Berl) 1999; 147:319-21.

(6) Parrott AC. Stress modulation over the day in cigarette smokers. Addiction 1995; 90:233-44.

(7) Perkins KA, Grobe JE. Increased desire to smoke during acute stress. Br J Addict 1992; 87:1037-40.

(8) Jarvis M. Smoking and Stress. In: Stephen A.Stansfeld, Michael G.Marmot, editors. Stress and the Heart: Psychosocial Pathways to Coronary Heart Disease.London: BMJ Books; 2002. p. 150-7. 
(9) Shiffman S, Hickcox M, Paty JA, Gnys M, Kassel JD, Richards TJ.

Progression from a smoking lapse to relapse: prediction from abstinence violation effects, nicotine dependence, and lapse characteristics. J Consult Clin Psychol 1996; 64:993-1002.

(10) Kabat-Zinn J. Full catastrophe living: how to cope with stress, pain and illness using mindfulness meditation. London: Piatkus; 1990.

(11) Grossman P, Niemann L, Schmidt S, Walach H. Mindfulness-based stress reduction and health benefits. A meta-analysis. J Psychosom Res 2004; 57:3543.

(12) Ussher M, West R, Doshi R, Sampuran AK. Acute effect of isometric exercise on desire to smoke and tobacco withdrawal symptoms. Hum Psychopharmacol 2006; 21:39-46.

(13) White G. Natural History and Antiquities of Selborne. London: Cassell And Company; 1908.

(14) Heatherton TF, Kozlowski LT, Frecker RC, Fagerstrom KO. The Fagerstrom Test for Nicotine Dependence: a revision of the Fagerstrom Tolerance Questionnaire. Br J Addict 1991; 86:1119-27.

(15) Pomerleau CS, Carton SM, Lutzke ML, Flessland KA, Pomerleau OF. Reliability of the Fagerstrom Tolerance Questionnaire and the Fagerstrom Test for Nicotine Dependence. Addict Behav 1994; 19:33-9. 
(16) West RJ, Russell MA. Pre-abstinence smoke intake and smoking motivation as predictors of severity of cigarette withdrawal symptoms.

Psychopharmacology (Berl) 1985; 87:334-6.

(17) West R, Hajek P. Evaluation of the mood and physical symptoms scale (MPSS) to assess cigarette withdrawal. Psychopharmacology (Berl) 2004; 177:195-9.

(18) West R, Ussher M, Evans M, Rashid M. Assessing DSM-IV nicotine withdrawal symptoms: a comparison and evaluation of five different scales. Psychopharmacology (Berl) 2006; 184:619-27.

(19) Daniel JZ, Cropley M, Fife-Schaw C. The effect of exercise in reducing desire to smoke and cigarette withdrawal symptoms is not caused by distraction. Addiction 2006; 101:1187-92.

(20) Daniel J, Cropley M, Ussher M, West R. Acute effects of a short bout of moderate versus light intensity exercise versus inactivity on tobacco withdrawal symptoms in sedentary smokers. Psychopharmacology (Berl) 2004; 174:320-6. 
Table 1 Mean ( \pm SD) demographic and smoking characteristics for the control and body scan groups

Control

$25.6 \pm 2.3$

$7.3 \pm 3.0$

$16.3+3.3$

$4.5 \pm 1.3$

$5.0 \pm 1.5$

$12.5 \pm 0.7$

Hours abstained

ECO following overnight abstinence

FTND $=$ Fagerström Test for Nicotine Dependence, $\mathrm{ECO}=$ expired carbon monoxide . 
Figure 1 (a-d). Ratings of MPSS withdrawal symptoms for the body scan and the control groups (baseline ratings subtracted from ratings taken immediately after intervention (0) and 5, 10 and 15 minutes post-intervention). 


\section{a) Strength of desire to smoke}

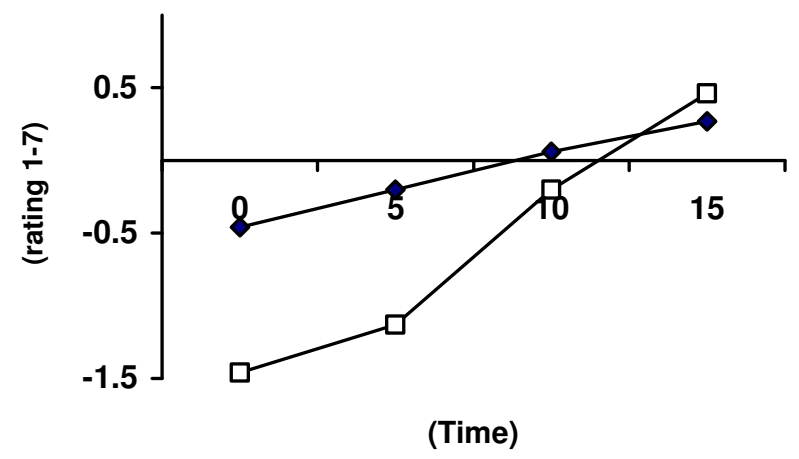

Group main effect $(\mathrm{F}=2.12, \mathrm{p}=0.15), \mathrm{ES}=.07$. Time main effect $(\mathrm{F}=36.10, \mathrm{p}<0.001), \mathrm{ES}=.54$. Group x Time $(\mathrm{F}=8.27, \mathrm{p}<0.001), \mathrm{ES}=.28$.

\section{c) Restlessness}

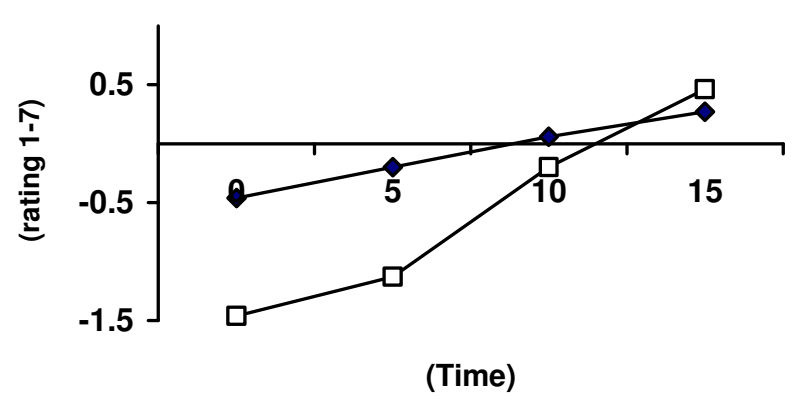

b) Irritability

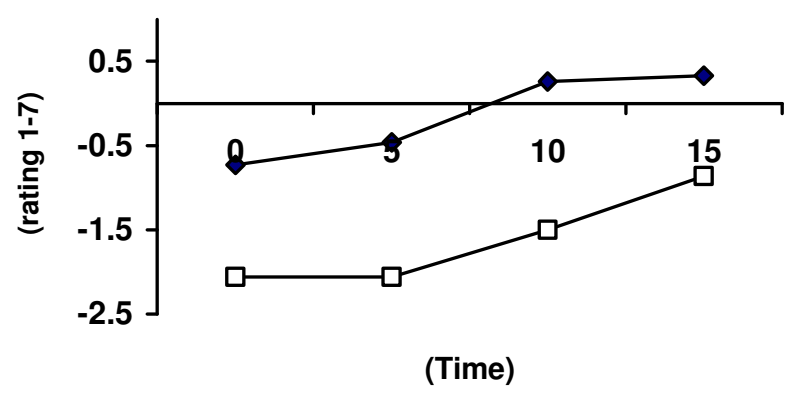

Group main effect $(\mathrm{F}=9.15, \mathrm{p}<0.005, \mathrm{ES}=.24$. Time main effect $(\mathrm{F}=35.89, \mathrm{p}<0.001), \mathrm{ES}=.56$. Group $\mathrm{x}$ Time $(\mathrm{F}=0.02, \mathrm{p}=0.83), \mathrm{ES}=.01$.

Group main effect $(\mathrm{F}=4.10, \mathrm{p}<0.05, \mathrm{ES}=.12$. Group main effect $(\mathrm{F}=9.55, \mathrm{p}<0.005), \mathrm{ES}=.25$. Time main effect $(\mathrm{F}=22.85, \mathrm{p}<0.001), \mathrm{ES}=.49$. Time main effect $(\mathrm{F}=11.72, \mathrm{p}<0.001), \mathrm{ES}=.29$. Group $x$ Time $(\mathrm{F}=0.61, \mathrm{p}=0.54), \mathrm{ES}=.02 . \quad$ Group $\mathrm{x}$ Time $(\mathrm{F}=1.88, \mathrm{p}=0.13), \mathrm{ES}=.06$.

$\mathrm{ES}=$ Effect Size

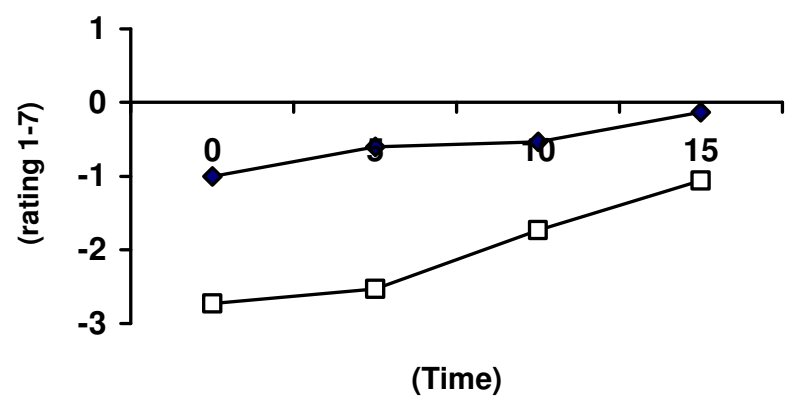

